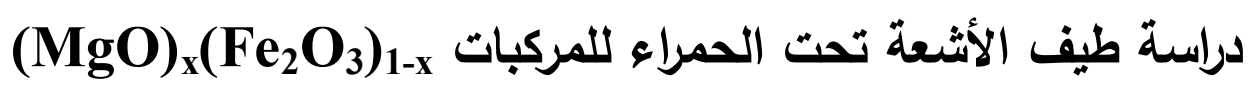

\author{
حذامه عبد محمد سليمان \\ قسم الفيزباء / كلية التربية \\ جامعة الموصل
}

القبول

$2012 / 02 / 01$
الاستلام

2011 / 09 / 28

\begin{abstract}
The Infrared spectroscopy in the frequency range $\left(400 \mathrm{~cm}^{-1}\right)$ to $\left(1000 \mathrm{~cm}^{-1}\right)$ of $(\mathrm{MgO})_{\mathrm{x}}\left(\mathrm{Fe}_{2} \mathrm{O}_{3}\right)_{1-\mathrm{x}}$ compounds at room temperature have been investigated. These compounds result from the solid state reaction of the $\mathrm{Fe}_{2} \mathrm{O}_{3}$ and $\mathrm{MgO}$ oxides at high temperatures around $\left(1000 \mathrm{C}^{\circ}\right)$. The infrared spectrum of these compounds show two main absorption bands; the first band, $v_{1}$ is caused by the stretching vibrations at the tetrahedral sites and the second band, $v_{2}$ is caused by the stretching vibrations at the octahedral sites of the lattice structure. Values of $v_{1}$ are higher than $v_{2}$. Moreover, the force constants of the bonds at the tetrahedral sites, $F_{\text {tet }}$ and octahedral sites, $F_{\text {oct }}$ have also been calculated. Values of $F_{\text {tet }}$ are higher than those of $\mathrm{F}_{\text {oct }}$. The results of this study agree quite well with the related studies for these compounds.
\end{abstract}

الخلاصة

تم في هذا البحث دراسة طيف الاثعة تحت الحمراء في مدى الترددات (1000 (400 (4)

الى (MgO) ${ }^{2}\left(\mathrm{Fe}_{2} \mathrm{O}_{3}\right)_{1-\mathrm{x}}$ الناتجة عن تفاعل

أوكسيد الحديد، ${ }^{2}{ }^{2} \mathrm{Fe}_{2} \mathrm{O}_{3}$ وكسيد المغنيسيوم، MgO والمحضرة بطريقة تفاعل الحالة الصلبة في

درجات حرارية عالية حوالي(

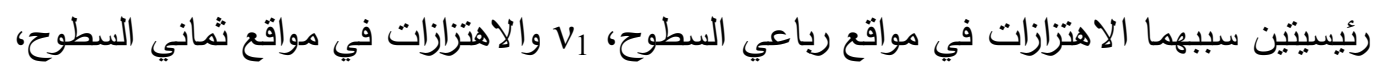
v2 2. كانت قيم حزم الامتصاص،

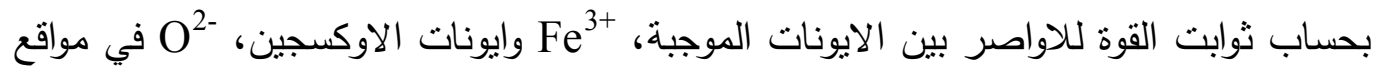

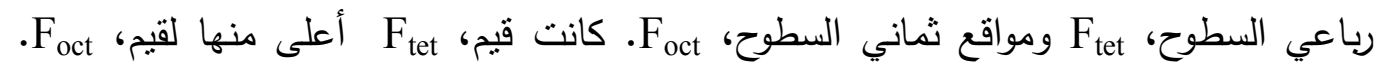
جاءت هذه النتائج مطابقة لنتائج الدراسات المشابهة لهذا النوع من المبات المركبات. 
المقدمة

أظهرت معظم التطبيقات التكنولوجية في مجالا التسجيل المغناطيسي بأنواعه المختلفة وصناعة المواد العازلة المستخدمة في اجهزة الاتصالات ذات الترددات المايكروية وحتى الترددات الراديوية وصناعة المغانط الكهربائية الدائمة وصناعة ذاكرة الحاسبات الإككرونية وفي الأجهزة الطبية ذات الدقة العالية، وغيرها من المجالات، أظهرت حاجتها الى مواد كهربائية ومغناطيسية ذات مواصفات معينة منل مقاومتها النوعية العالية وقيمة منخفضة لفقدان للتيارات الدوامة. ومن اهم المواد التي يتوافر فيها هذه الخصائص هي المواد المغناطيسية المعروفة بالفريتات أو الفرايت (Ferrites)]1-2]. ان اساس الخاصية المغناطيسية لهذه المواد هو عدم تكافؤ العزوم المغناطيسية في مواقع الثبيكة المختلفة حيث يكون فيها العزوم المغناطيسية غير متكافئة مما ينتج عنه الخاصية الفيريمغناطيسية (Ferrimagnetism). تمتلك هذه الخاصية المغناطيسية عدد كبير جدا من المركبات ذات الصيغة الكيميائية TMO.Fe $2 \mathrm{C}_{3}$ إذ يمثل TM عنصر انتقالي ثنائي التكافؤ مثل المغنيسيوم، Mg والزنلك، Zn والنيكل، Ni والكوبلت، Co غيرها]

يتمثل التركيب البلوري لهذه المركبات بما يعرف بتركيب السبينيل. تحتوي وحدة الخلية لهذا التركيب على 56 أيون منها 32 أيون أوكسجين، مشكلّة شبيكة مكعبة محكمة الرص A Aمركزة الأوجه. يتميز في هذا التركيب مجموعتان من المواقع: المجموعة الأولى تسمى مواقع وكل منها يحاط بأربع أيونات أوكسجين وتعرف أيضا بمواقع رباعي السطوح ( Tetrahedral Sites والمجموعة الثانية تسمى مواقع B وكل منها يحاط بست أيونات أوكسجين وتعرف ايضا بمواقع ثماني السطوح (Octahedral Sites).

هنالك نوعان من تركيب السبينيل[3]: السبينيل الطبيعي (Normal Spinel): والذي نتخل فيه كل أيونات العنصر الانتقالي ثنائي التكافؤ، TM مواقع رباعي السطوح بينما تشغل أيونات الحديد ثلاثية التكافؤ Fe مواقع ثماني

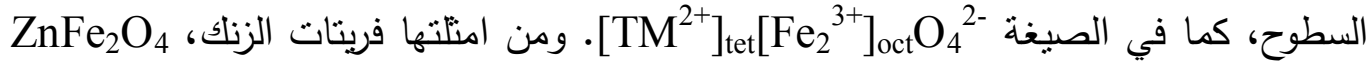
وفريتات الكادميوم، مثل أيونات الزنك وأيونات الكادميوم تقضل مواقع رباعي وتحتل أيونات الحديد ثماني السطوح[4] - ت والسبينيل المعكوس (Inverse Spinel): والذي تشغل فيه أيونات TM مواقع رباعي السطوح بينما تتوزع أيونات الحديد Fe بالتساوي بين مواقع رباعي السطوح ومواقع ثماني السطوح. كما

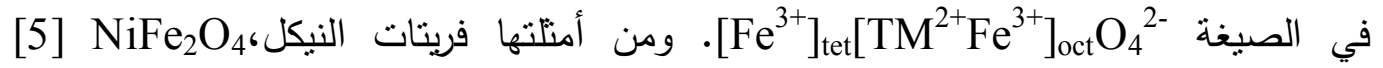


والنحاس، العديد من الدراسات ان الأيونات الانتقالية ثنائية التكافؤ مثل أيونات النيكل والنحان النحاس والكوبلت

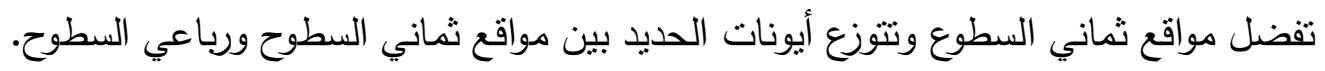

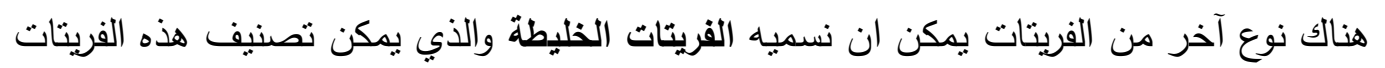

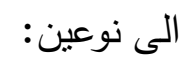

النوع الأول: والذي يضاف فيه عنصران او أكثر من العناصر الانتقالية والتي يمكن تمثيل تركييها الكيميائي بالصيغة الكيميائية الاتية:. TMFe $2 \mathrm{O}_{4}$ (TM=Mg, Cu, Zn

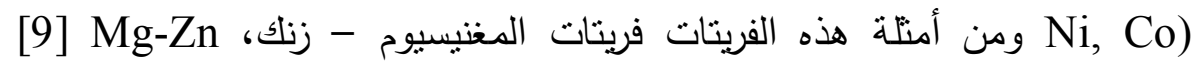
وفريتات النيكل-نزنك، Ni-Zn [10] وغيرها. النوع الثاني: من الفرينات الخليطة هي الفريتات التي يستعاض فيها عن بعض ذرات الترات الحديد ثلاثية التكافؤ بذرات ثلاثية النكافؤ ولكنها غير مغناطيسية مثل عنصر الالمنيوم

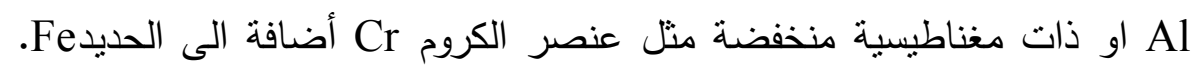

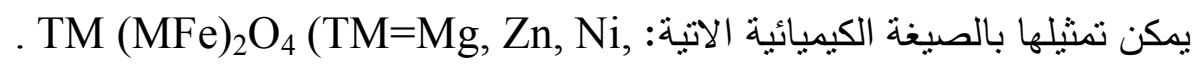

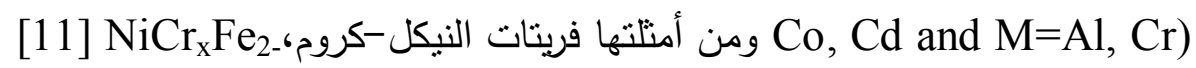

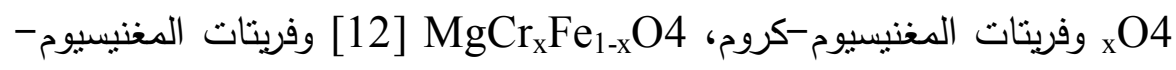
المنيوم-كروم تركيب السبينيل الا ان خصائصها الكهبائية والمغناطيسية قد تاثرت بشكل كبير .

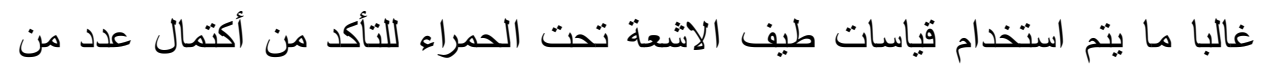

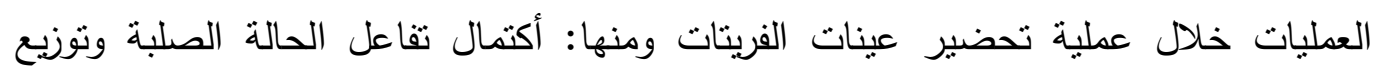
الأيونات الموجبة في التركيب البلوري وتتوه تكوين تركيب السبينيل المكتمل (التام) وترتيب الأيونات الموجبة وقياس قيم ثوابت القوة في مواقع رباعي وثماني السطوح في هذه المبردي المركبات.

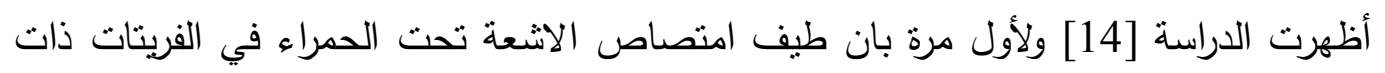

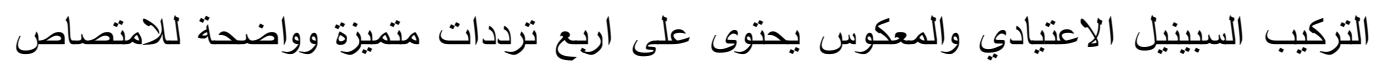

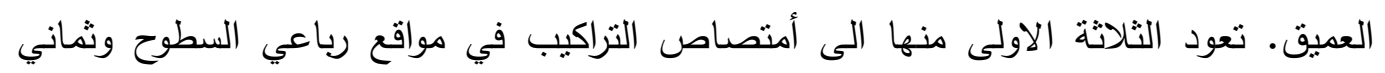
السطوح بينما يعزى التردد الرابع الى بعض ترددات الثبيكة للايونات الموجبة في مواقع رباعي في لئي

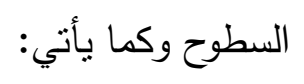
الحزمة الأولى سبيها الترددات في مواقع A وهي الآصرة

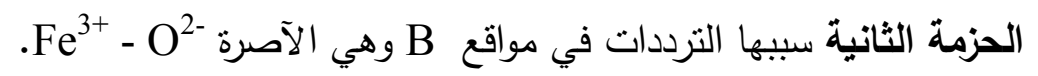

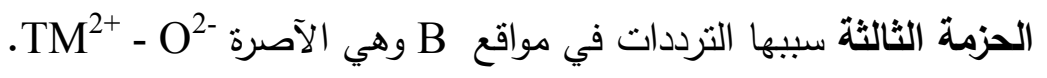


الحزمة الرابعة سببها الترددات في مواقع ثماني السطوح للاواصر اذ تمثل TM TM TM $^{2+} \mathrm{O}^{2-}$ الحزمة على كتلة العنصر الانتقالي ثنائي التكافؤ.

ان الهذف من هذه الدراسة هو القيام بتحضير عينات فريتات المغنيسيوم بخلط مركبات

اوكسيد الحمراء في للعينات الناتجة. من الجدير بالذكر انه تم دراسة خاصيتي التوصيلية الكهربائية والقدرة الكهروحراية وطيف حيود الاشعة السينية لهذه العينات. ان تفاصيل هذه الدراسة منشورة

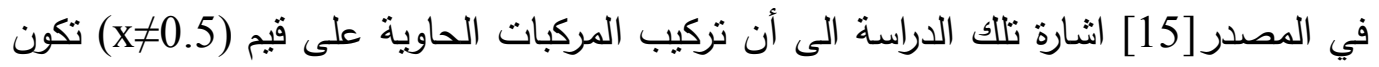
مختلفة بعض الثيء عن العينة الحاوية على (x=0.5) ويعزى هذا الى احتوائهما على بقايا من أوكسيد الحديد هذه العينات على أطوار ثنانوية أخرى اكثر تعقيدا.

\section{تحضير العينات}

تم تحضير عينات فريتات المغنيسيوم بخلط مركبات اوكسبد وحسب النسب المثبته في المركب. تم سحق هذه الاكاسيد سوية في بوتقة العقيق

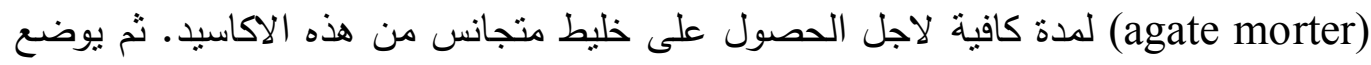
خليط هذه الاكاسيد لكل مركب في الفرن الكهربائي ويتم تسخينها لمدة 24 ساعة عند درجة حرارة

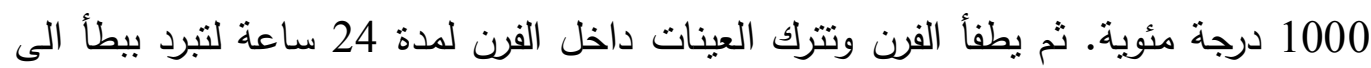
درجة حرارة الغرفة. ثم يعاد سحق المركبات المذكورة لمدة كافية لرفع درجة التجانس قدر درد الامكان. ثم توضع في الفرن وترفع درجة حرارتها الى 1100 درجة مئوية لمدة 24 ساعة اخرى. ثم يطفأ الفرن وتترك العينات داخل الفرن لددة 24 ساعة لتبرد ببطأ الى درجة حرارة الغرفة. يعاد سحق المركبات الناتجة لغرض تهيئة العينات لقياسات الاثعة تحت الحمراء بخلط

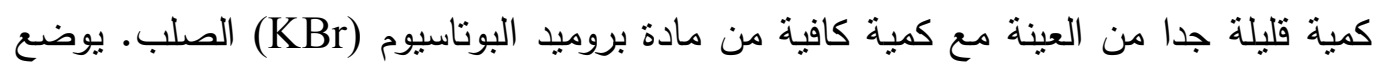

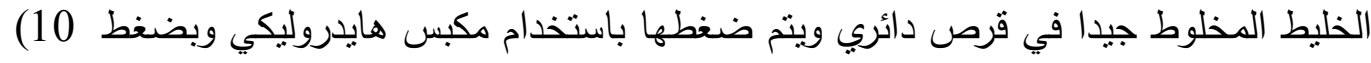
tons/cm²)

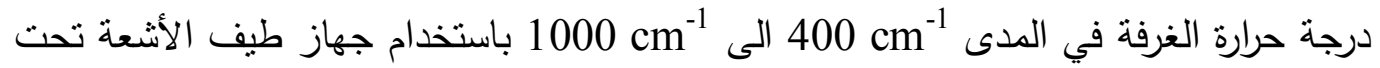

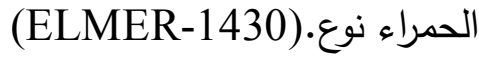

النتائج وإلمناقشة

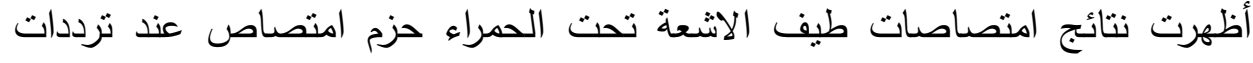

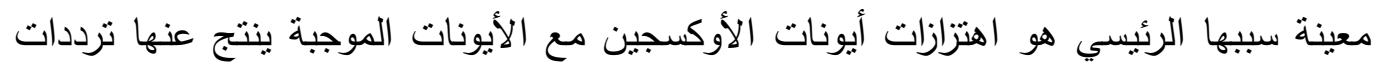

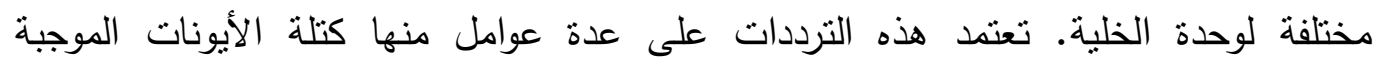


ومعاملات الشبيكة وأواصر الأوكسجين-الأيون الموجب وغيرها. يوضح الثكل (1) والجدول (1) طيف امتصاص الاشعة تحت الحمراء للعينات المدروسة في مدى الترددات 1000-400 1000 cm

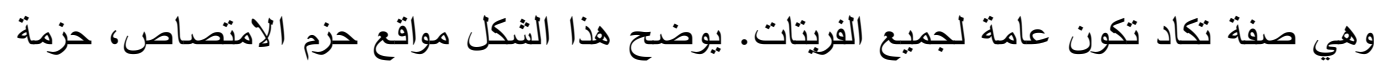

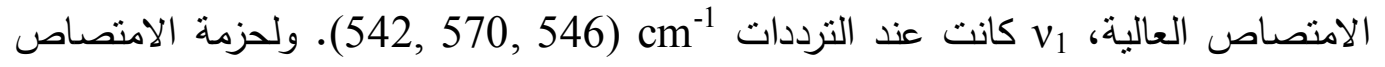
الواطئة، $v_{2}$ كانت عند الترددات المغنيسيوم بنسبة (0.75, 0.50, 0.25) على التوالي. تنفق هذه القيم مع قيم الترددات لفريتات

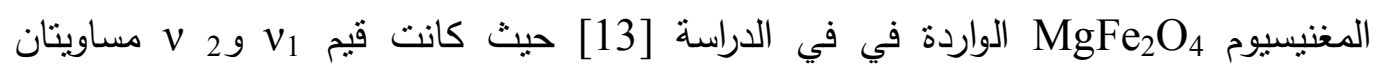
الى v مساويتان الى لى $v_{2}$ المختلفة الى اختلاف طرائق التحضير والمعالجة الحرارية ذات التأثثر البالغ على طبيعة المادة

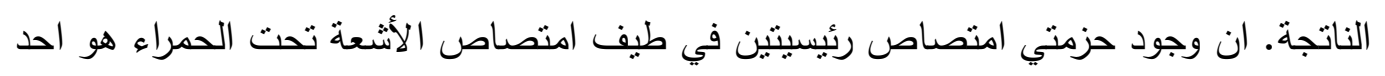
الادلة العملية على وجود تركيب السبينيل التام الذي يمتلك الثبيكتين الفرعيتين (sublattices):

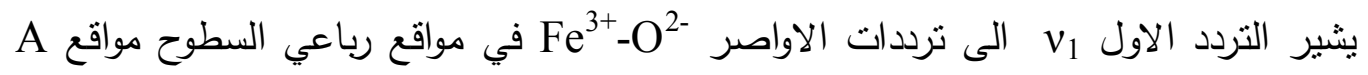

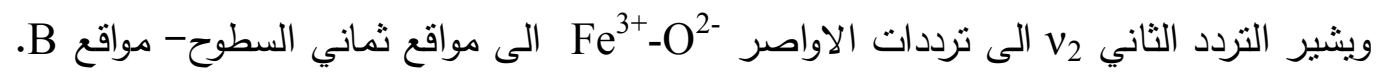

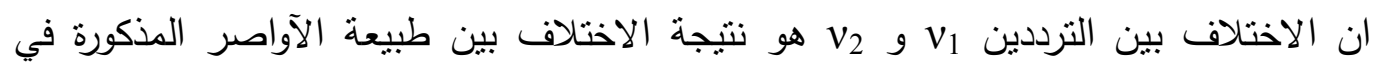

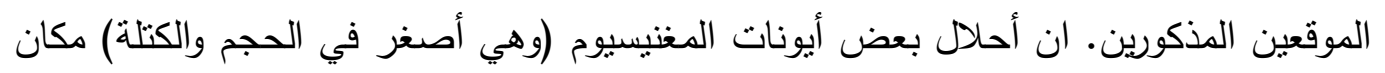

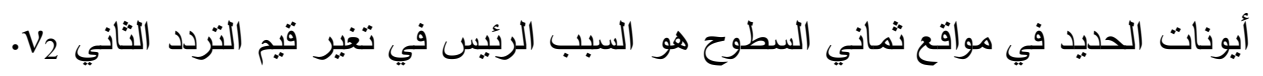

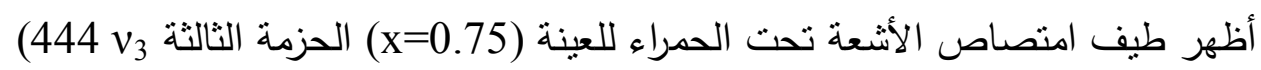

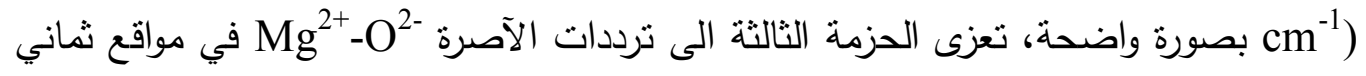

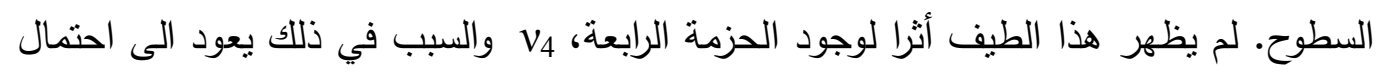

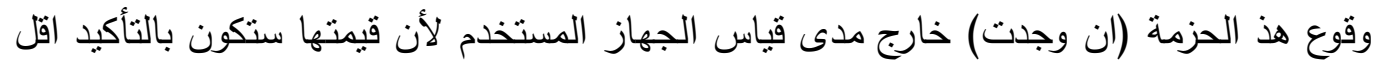

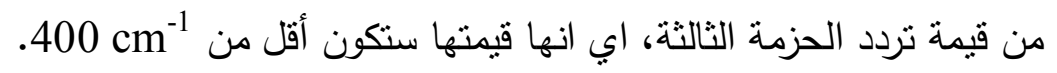

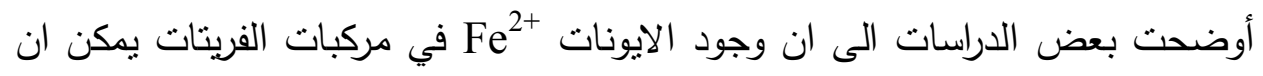

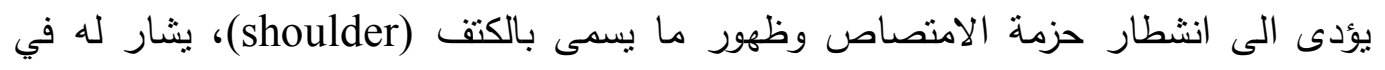
بعض الاحيان بالتزدد الخامس v. ان وجود متل هذه الايونات يسبب نتوها تركيبيا موضعيا

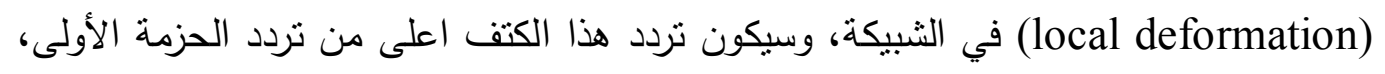

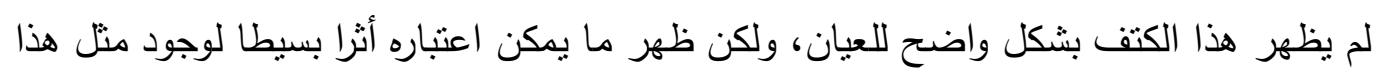

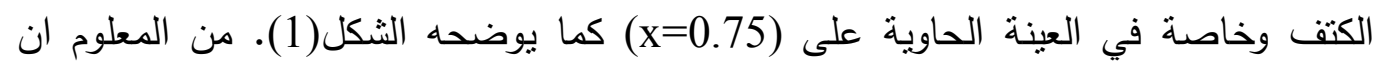
أيونات المغنيسيوم تفضل مواقع ثماني السطوح بنسبة تزيد على 
الايونات الأقل من \% 10 مواقع رباعي السطوح. ان اضافة ايونات المغنيسيوم واحتلالها

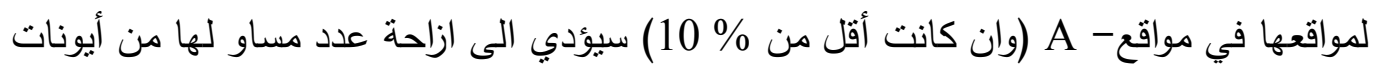

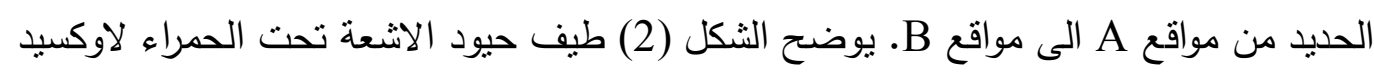

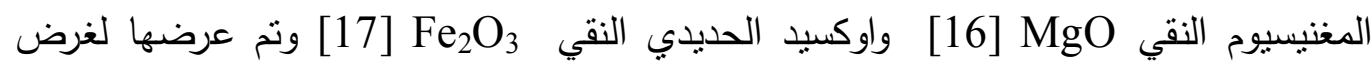

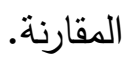

أوضحت نتائج هذه الدراسة الى ان العينة الحاوية على (x=0.5) اظهرت حزمني امتصاص تامتنن واكثر وضوحا من العينتين الباقيتين وهذه احدى خصائص الفريتات ذات

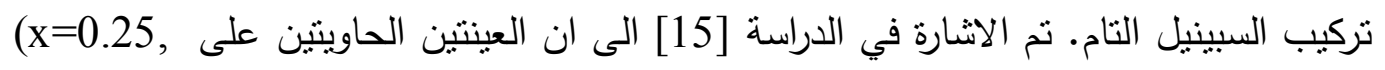
(0.75 قد تحتويان على بقايا من اوكسيد المغنيسيوم واوكسيد الحديد غير تامة التفاعل وان التركيب الحاوي على (x=0.50) هو الاقرب الى تركيب السبينيل التام. وهذه ما ايدته هذه

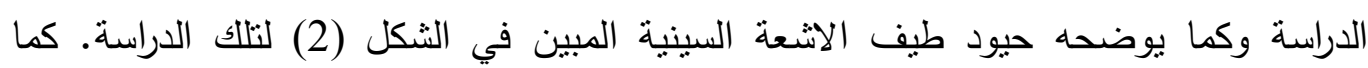

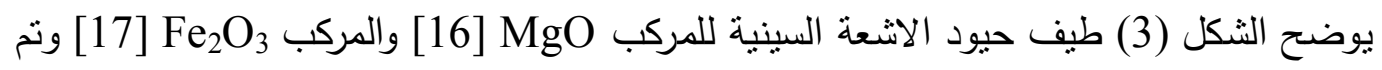
عرضها لغرض المقارنة.

يمكن حساب ثنابت القوة Force constant) للمواقع رباعي السطوع Fet Feردات

$$
\begin{aligned}
& \text { وثماني السطوح Foct للترددات } \\
& F_{\text {tet }}=4 \pi^{2} c^{2} v_{1}^{2} \mu \\
& F_{\text {oct }}=4 \pi^{2} c^{2} v_{2}^{2} \mu
\end{aligned}
$$

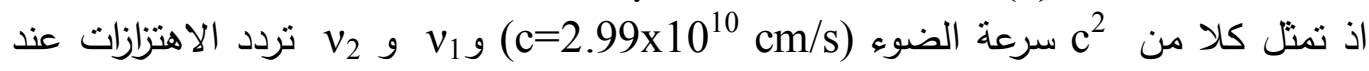

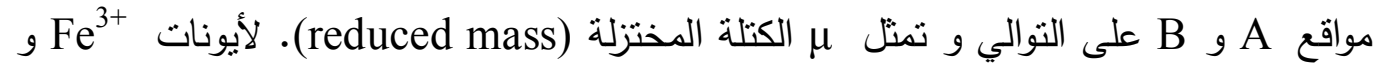
$\mathrm{O}^{2-} \cdot[6]$ في البداية يجب حساب قيمة بر السماوية الى (6)

$$
\mu=\left(m_{1} * m_{2}\right) /\left(m_{1}+m_{2}\right)
$$

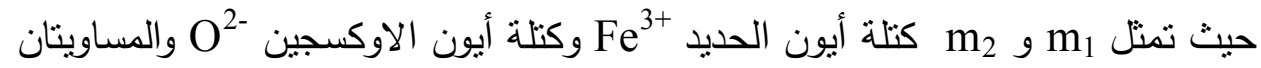

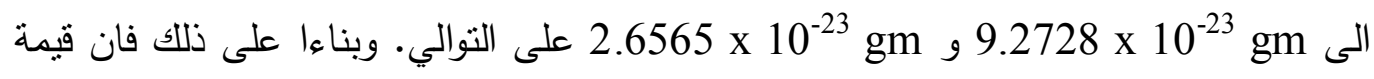

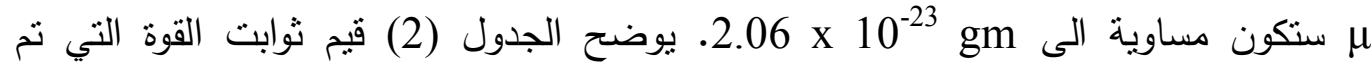

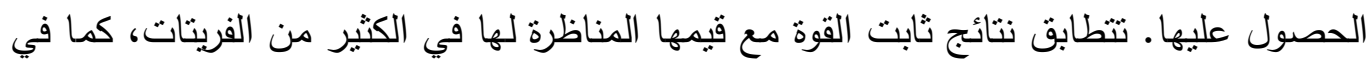
فريتات النحاس-زنك [6] وفريتات النيكل-زنكك المطعمة بالكوبلت [18]. تتطابق هذه النتائج مع نتائج دراسات طيف الآثعة تحت الحمراء المشابهة للعينات

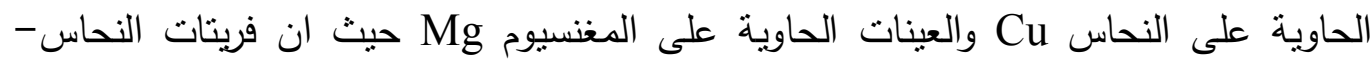
الحديد تتثابه الى حد كبير مع فريتات المغنسيوم-حديد اذ كلاهما يكوّن اسبينيل معكوس.

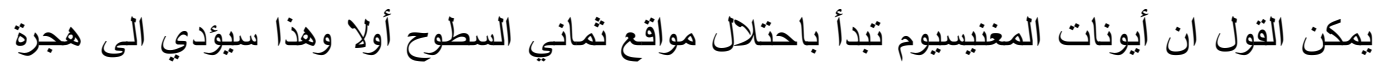

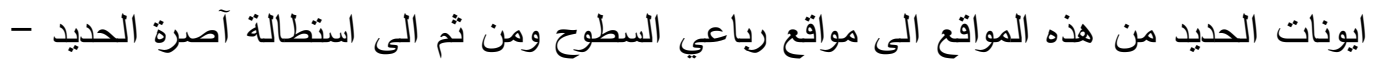


أوكسجين وانخفاض في عدد الموجة للحزمة. ينعكس هذا التأثير في التراكيز العالية لأيونات المغنيسيوم حيث سيتحول الأحلال من مواقع رباعي السطوح الى مواقع ثماني السطوح، اي

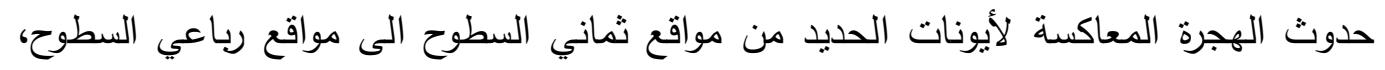

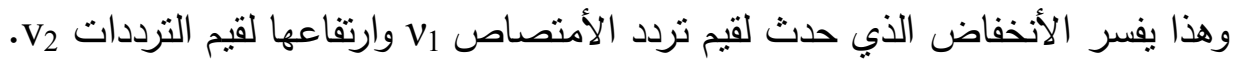

أظهرت نتائج دراسة طيف امتصاص الأشعة تحت الحمراء للمركبات في مدى الطيف (MgO) $\left(\mathrm{Fe}_{2} \mathrm{O}_{3}\right)_{1-\mathrm{x}}$

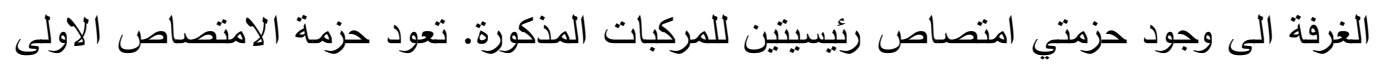
(العالية) الى الترددات الواقعة في مواقع رباعي السطوح وتعود حزمة الامتصاص الثانية (الواطئة) الى الترددات الواقعة في مواقع ثماني السطوح في الشبيكة. امتلكت حزمة الترددات

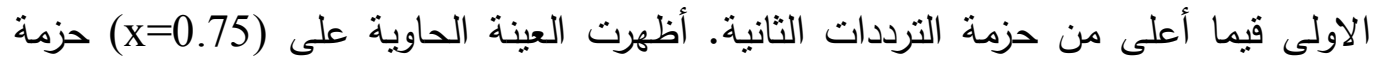
ثالثة. لم تظهر هذه العينات أثرا للحزمة الرابعة لوقوعها خارج نطاق مدى قياس التهات الجهاز

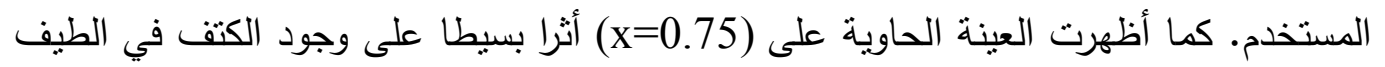
عند تردد أعلى من الحزمة الاولى. تم استخدام نتائج حيود الاشعة تحت الحمراء في حساب قيم المباء ثوابت القوة للاواصر بين الايونات الموجبة والسالبة في مواقع رباعي السطوح ومواقع ثماني

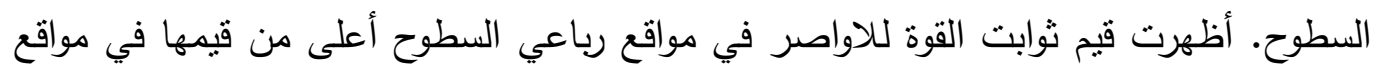
ثماني السطوح.

\section{المصادر}

1) Snelling E. C. (Ed.) 1988, "Soft Ferrites, Properties and Applications", 2nd ed., Butter Worth and Co. (Publisher) Ltd. London, (and references therein).

2) A. Goldman "Modern Ferrite Technology", 2nd Ed. Springer Science \& Business Media, Inc. (2006).

3) K. J. Standley, "Oxide Magnetic Materials", 2nd Ed. Clarendon Press Oxford (1972).

4) M. Chakrabarti, D. Sanyal and A. Chakrabarti, "Preparation of $\mathrm{Zn} 1-\mathrm{xCdxFe} 2 \mathrm{O} 4(\mathrm{x}=0.0,0.1,0.3,0.5,0.7$ and 1.0) ferrite samples and their characterization by Mossbauer and positron annihilation techniques" J. Phys: Condensed Matter, Vol. 19 (2007) 1-11.

5) Kinemuchi Y., Ishizaka K., Suematsu H., Jiang W., Yatsui K. "Magnetic properties of nanosize NiFe2O4 particles synthesized by pulsed wire discharge". Thin Solid Films 407 (2002) 109-113.

6) Zaki H. M. and Dawoud H. A. "Far-Infrared Spectra for Copper-Zn mixed Ferrites". Physica B, 405 (2010) 4476-4479.

7) Lee J. G., Park J. Y., Oh Y-J and Kim C. S., "Magnetic Properties of CoFe2O4 thin films prepared by a sol-gel method" J. Appl. Phys. 84 (1998) 2801-2805. 
8) Turkin A. I. and Drebushchak V. A., Synthesis and Calorimetric Investigation of Stoichiometric Fe-spinels: MgFe2O4, J. Cryst. Growth. 205 (2004), 165-167.

9) Ladgaonkar B. P., Vasambekar P. N. and Vaingankar A. S., Effect of $\mathrm{Zn} 2+$ and $\mathrm{Nd} 3+$ Substitution on Magnetism and $\mathrm{AC}$ Susceptibility of Mg ferrite, J. Magn. Magn. Mater. 210 (2000), 289-294.

10) Ghazanfar U., Siddiqi S. A. and Abbas G. "Study of room temperature dc resistivity in comparison with activation energy and drift mobility of Ni-Zn Ferrites". Mater. Sci. Eng. B118 (2005) 132-134

11) A. M. Gismelseed and A. A. Yousif, Mossbauer study of chromium-substituted nickel ferrites. Physica B. Vol. 370 (2005) 215-222.

12) P. P. Hankare, V. T. Vader, U. B. Sankpal, L. V. Gavali, R. Sasikala and I. S. Mulla. "Effect of sintering temperature and thermoelectric power studies of the system $\mathrm{MgFe} 2-\mathrm{xCrxO} 4$ ". Solid State Scienc. Vol. 11 (2009) 2075-2079.

13) Thummer K. P., Pandya M. P., Jani K. H., Modi K. B. and Joshi H. H., "Microscopic and Macroscopic Magnetic Properties of the MgAlxCrxFe2-2xO4 Spinel Ferrite System". Materials Science Vol. 40 No. 1 (2004).

14) R. D. Waldron, "Infrared Spectra of Ferrites", Phys. Rev. Vol. 99 (1955) 1727-1735.

15) Mohammed K. A. and Mohammed H. A. Electrical Conductivity and Thermoelectric Power of Iron-Magnesium $(\mathrm{MgO}) \mathrm{x}(\mathrm{Fe} 2 \mathrm{O} 3) 1-\mathrm{x}$ Compounds. J. Abhath Al- Yarmouk, Vol. 19, No. (1) (2010) 1-14.

16) T. Lopez, I. Garcia-Cruz and R. Gomez, "Synthesis of magnesium oxide by the Sol-Gel method: Effect of the PH on the surface hydroxylation". J. Catal. Vol.(127 (1991) 75-85.

17) N. Du, Y. Xu and H. Zhang, "Selective synthesis of Fe2O3 and Fe3O4 Nanowires via a single precursor: A general method for metal oxide nanowires", Nanoscale Res. Lett. Vol.5 (2010) 12951300 .

18) M.A. Amer, A. Tawfik, A.G. Mostafa, A.F. El-Shora, S.M.Zaki "Spectral Studies of Co substituted Ni-Zn Ferrites". J. Magn. Magn. Mater. Vol.323 (2011) 1445-1452. 
حذامه عبد محمد سليمان

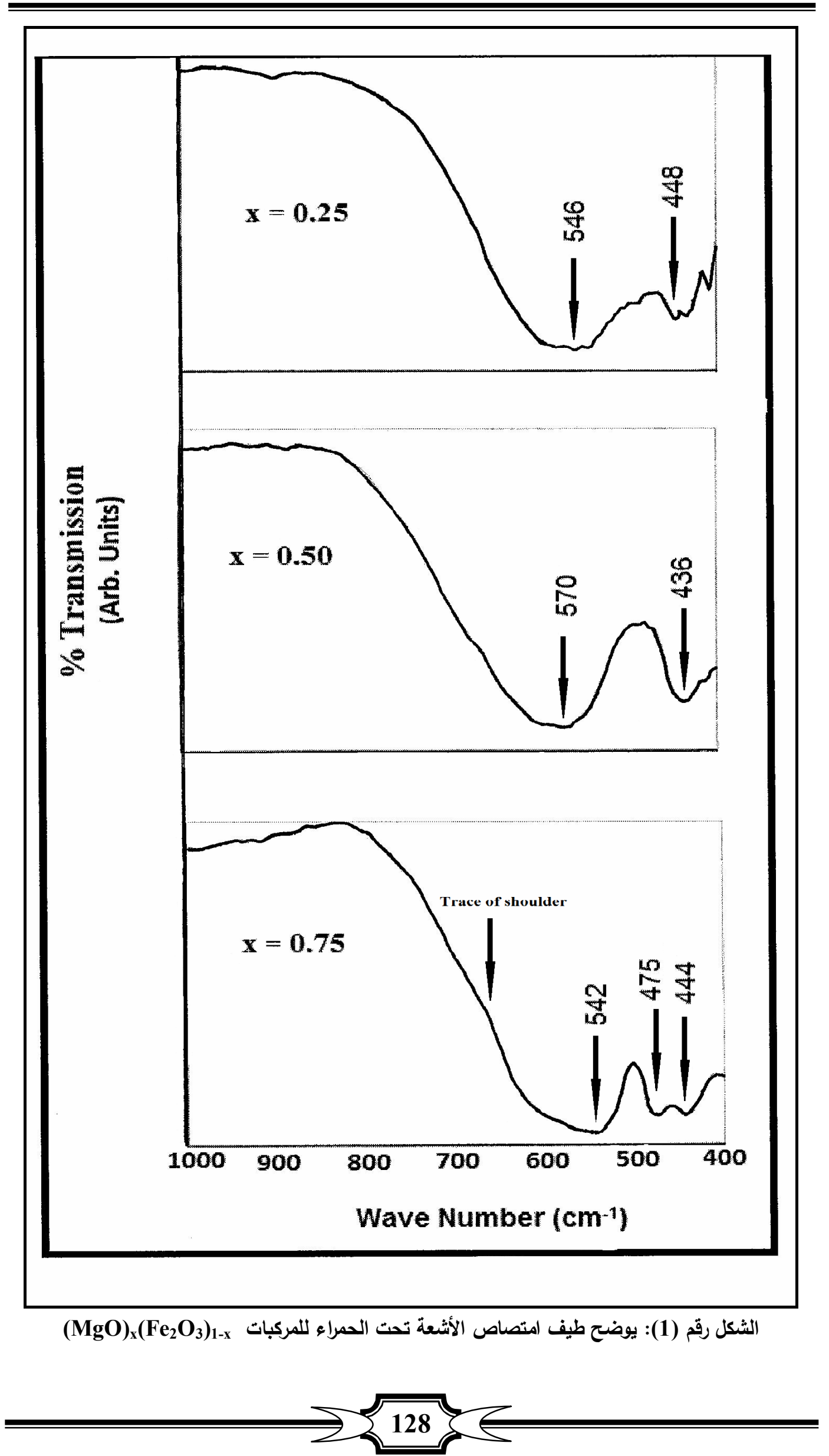




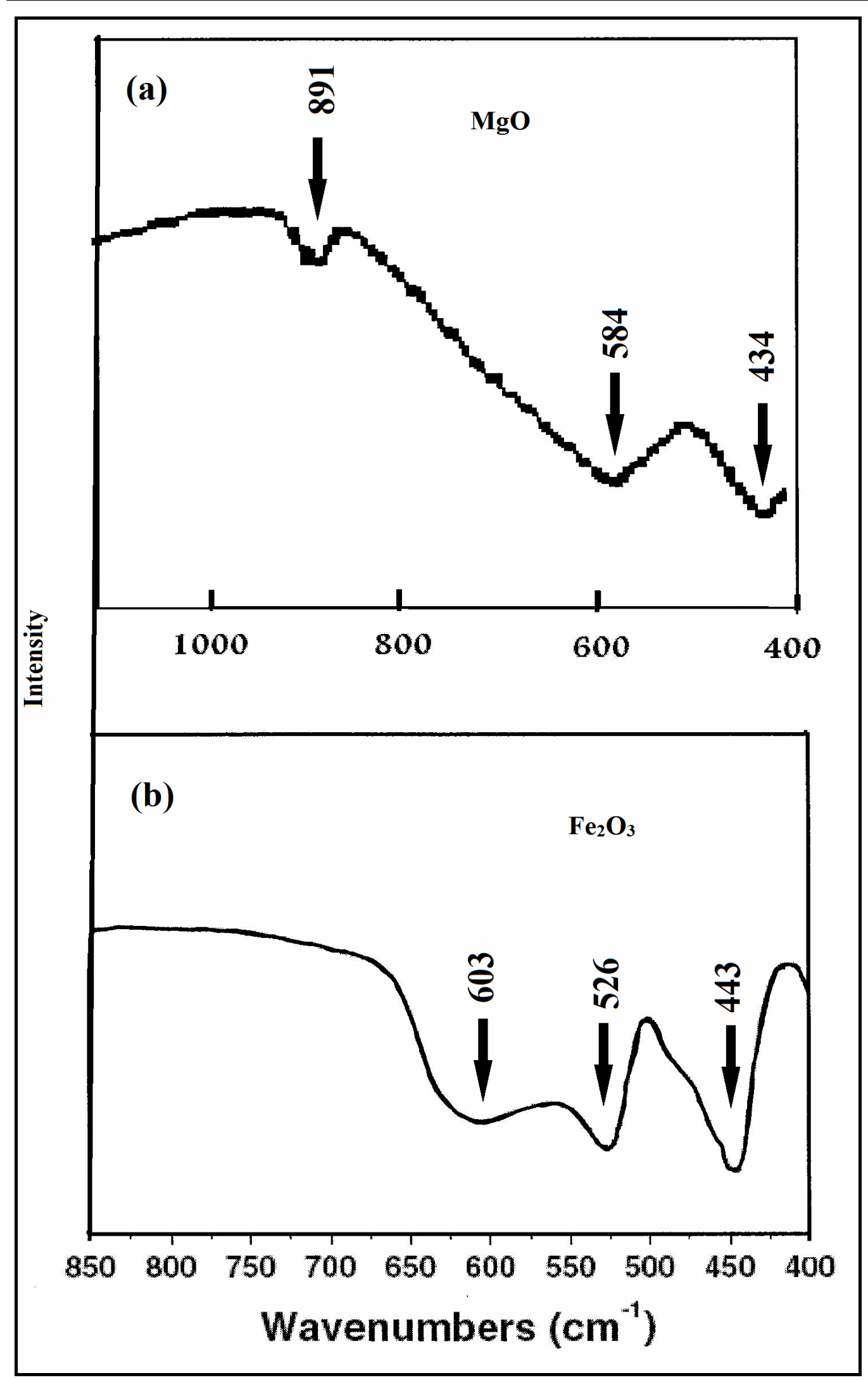

الثكل رقم (2): يوضح طيف امتصاص الأشعة تحت الحمراء:

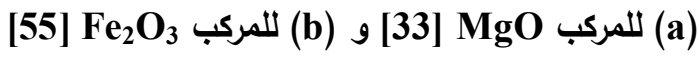


حذامه عبد محمد سليمان

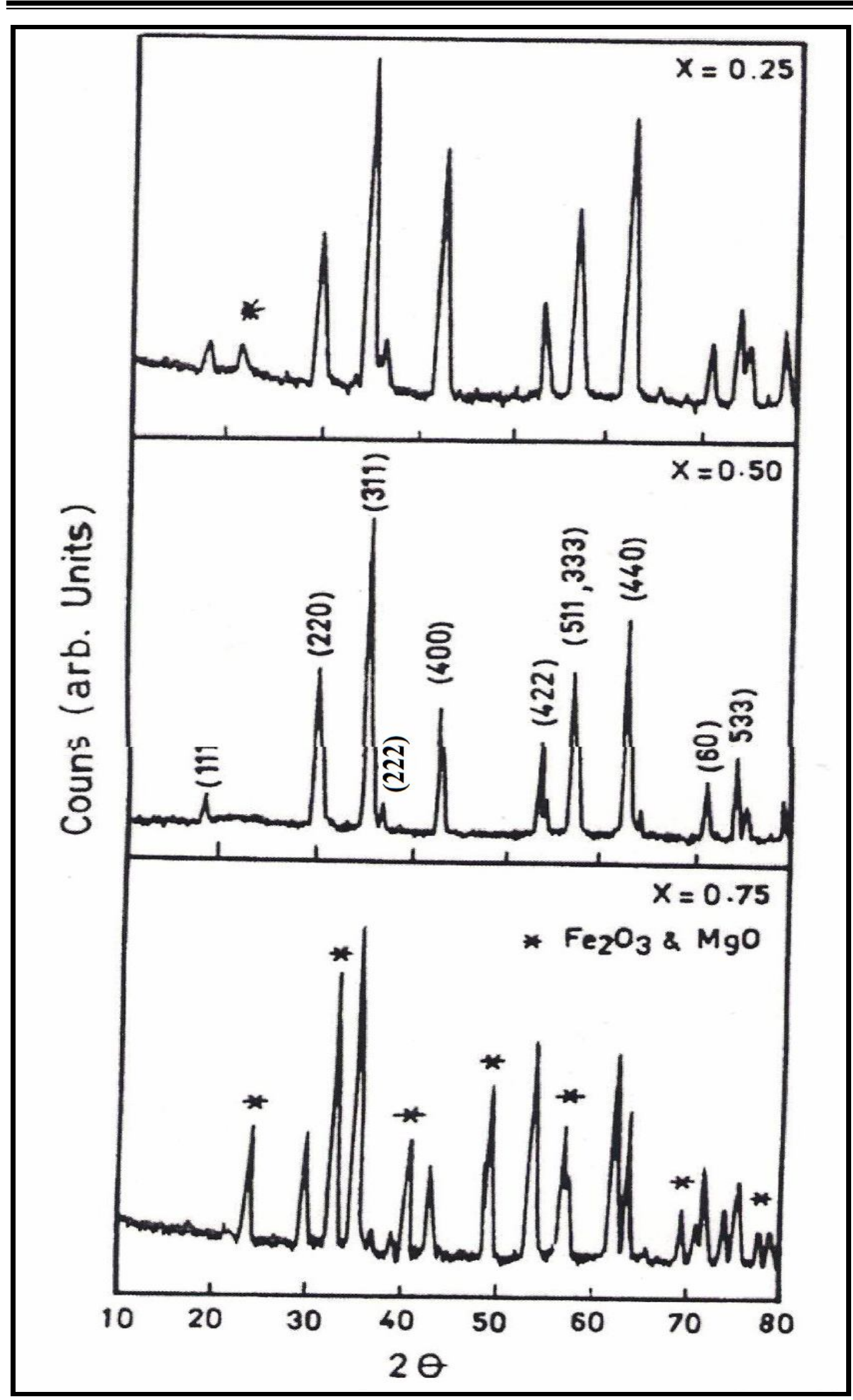

الثكل رقم (3): يوضح طيف حيود الأثعة السينية للمركبات .

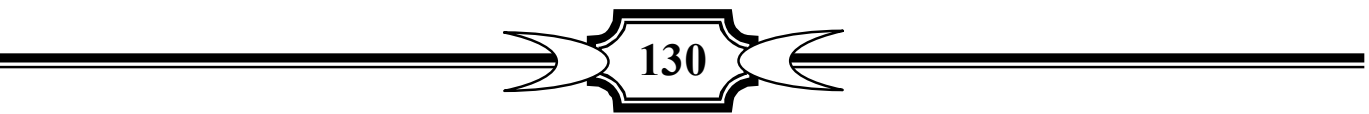



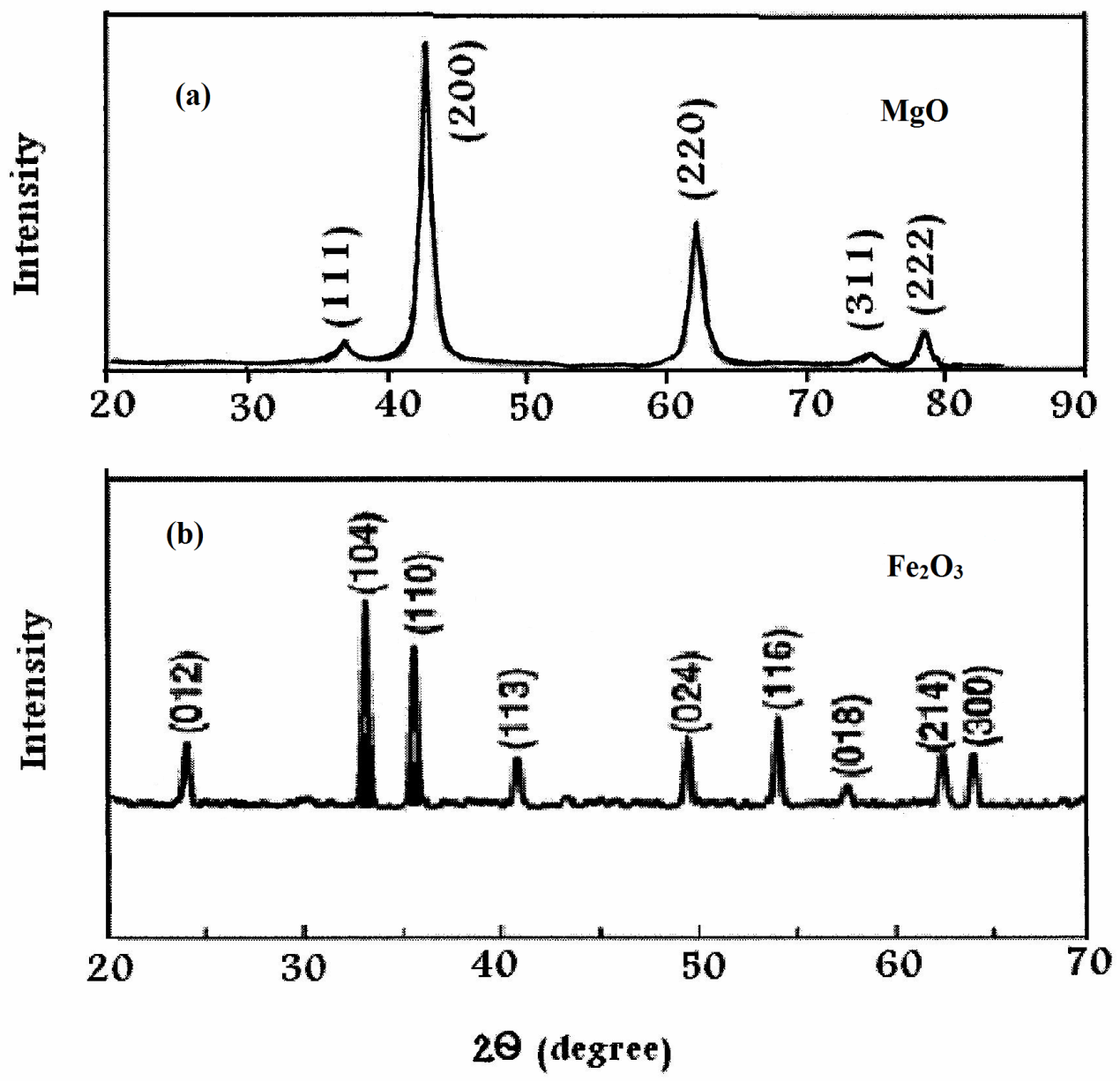

الشكل رقم (4): يوضح طيف حيود الأشعة السينية:

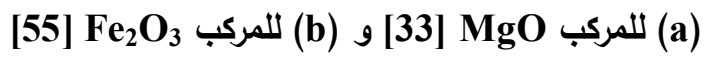

\begin{tabular}{|c|c|c|c|}
\hline $\mathbf{x}$ & $\begin{array}{c}\mathbf{v}_{\mathbf{1}} \\
\left(\mathbf{c m}^{-\mathbf{1}}\right)\end{array}$ & $\begin{array}{c}\mathbf{v}_{\mathbf{2}} \\
\left(\mathbf{c m}^{-\mathbf{1}}\right)\end{array}$ & $\begin{array}{c}\mathbf{v}_{\mathbf{3}} \\
\left(\mathbf{c m}^{\mathbf{- 1}}\right)\end{array}$ \\
\hline 0.25 & 546 & 448 & ---- \\
\hline 0.50 & 570 & 436 & ----- \\
\hline 0.75 & 542 & 475 & 444 \\
\hline
\end{tabular}

الجدول رقم (1): يوضح قيم أمتصاص الأشعة تحت الحمراء للمركبات

\begin{tabular}{|c|c|c|}
\hline $\mathbf{x}$ & $\begin{array}{c}\mathbf{F}_{\text {tet }} \\
\mathbf{d y n} / \mathbf{c m} \\
(\mathbf{x ~ 1 0} \mathbf{~ 5})\end{array}$ & $\begin{array}{c}\mathbf{F}_{\text {oct }} \\
\mathbf{d y n} / \mathbf{c m} \\
\left(\mathbf{x ~ 1 0} \mathbf{~ 1 0}^{\mathbf{5}}\right)\end{array}$ \\
\hline 0.25 & 2.166 & 1.458 \\
\hline 0.50 & 2.361 & 1.381 \\
\hline 0.75 & 2.135 & 1.640 \\
\hline
\end{tabular}

الجدول رقم (2): يوضح قيم ثوابت القوة للمركبات 